\title{
Evaluación de dos pruebas comerciales para el serodiagnóstico de la tuberculosis pulmonar
}

\author{
Adriana D'Alessandro y Jacobus $H$. de Waard
}

\section{Evaluation of two commercial tests for the serodiagnosis of pulmonary tuberculosis}

Two commercial serological tests, Pathozyme- TB complex plus ${ }^{\mathrm{TM}}$ and Pathozyme-Myco IgG ${ }^{\mathrm{TM}}$, were evaluated for their sensitivity and specificity in HIV negative patients with serum samples from pulmonary tuberculosis (TB) patients and tuberculin positive, healthy controls. The sensitivity for the two tests was 50 and $80 \%$ respectively and the specificity 95 and $80 \%$. With a prevalence of pulmonary TB of $10 \%$ in symptomatic patients who consult our laboratory, the positive predictive values (PPV) for the two ELISA tests were 92 and 80\%, and the negative predictive values (NPV) 98 and 80\% respectively. In the same patient group, the AFB smear has a PPV of $100 \%$ and a NPV of $97 \%$. We conclude that AFB smear is a better mass screening tool than ELISA in symptomatic patients who consult our laboratory. The test could be useful as a complementary test in excluding or confirming TB in selected, smear negative, symptomatic patients with high degree of clinical suspicion of active TB.

Key words: Pulmonary tuberculosis, serodiagnosis, Pathozyme- TB complex plus, Pathozyme-Myco IgG. Palabras clave: Tuberculosis pulmonar, serodiagnóstico, Pathozyme-TB complex plus, Pathozyme Myco IgG.

\section{Introducción}

$\mathrm{P}$ ara el control de la tuberculosis (TBC), es crucial un diagnóstico rápido, específico y sensible de la TBC pulmonar, la forma contagiosa de la enfermedad. Un diagnóstico retrasado o falso negativo contribuye a la transmisión de Mycobacterium tuberculosis y por lo tanto, al incremento de la morbilidad y mortalidad por la enfermedad. El diagnóstico bacteriológico generalmente se lleva a cabo a través de la baciloscopia y el aislamiento de $M$. tuberculosis por cultivo. La baciloscopia es económica, fácil de implementar y proporciona resultados rápidos. Sin embargo, posee baja sensibilidad: 30 a $40 \%$ de los pacientes con TBC pulmonar cursan con baciloscopia negati$\mathrm{va}^{1,2}$. El cultivo es más sensible en el diagnóstico y, en general, se diagnostican con él hasta $40 \%$ más muestras positivas. Sin embargo, la desventaja es que el diagnóstico por cultivo tarda 3 a 6 semanas antes de dar un resultado positivo. Además, especialmente en países en desarrollo, muchos laboratorios no son aptos para el cultivo de micobacterias por falta de bioseguridad, personal entrenado y carencia del medio de cultivo específico que se requiere para su aislamiento.

Por esta razón, fueron desarrolladas pruebas alter- nativas, entre ellas las pruebas serológicas, de las que especialmente el ensayo de ELISA podría tener grandes ventajas para el diagnóstico de TBC. Son fáciles para ejecutar y la técnica, en general, está en uso en laboratorios clínicos, no siendo necesario efectuar nuevas inversiones en entrenamiento de personal o equipamiento. En adición, la prueba es costo-efectiva y el resultado está rápidamente disponible. Numerosas pruebas serológicas que usan varios antígenos fueron desarrolladas ${ }^{3-6}$ y varias están disponibles comercialmente en este momento. En un estudio comparativo, se evaluaron siete pruebas serológicas de cuatro casas comerciales para determinar la sensibilidad y especificidad en el diagnóstico de TBC pulmonar ${ }^{7}$. Se mostró en ese estudio que el ensayo de ELISA Myco IgG®, tenía la sensibilidad más alta (58\%). La especificidad de esta prueba determinada con suero de pacientes sanos y tuberculina positiva, fue de $89 \%$. En el mismo estudio, otro ensayo de ELISA de la misma casa comercial, TB complex plus ${ }^{\circledR}$, mostró una sensibilidad de sólo 15\% en pacientes con TBC pulmonar pero una especificidad más alta, 97\%, en los controles sanos con tuberculina positiva.

El presente trabajo evalúa la sensibilidad y especificidad de estos dos ensayos de ELISA en el diagnóstico
Laboratorio de Tuberculosis, Instituto de Biomedicina, Caracas, Venezuela

Recibido: 15 de noviembre de 2006 Aceptado: 01 de noviembre de 2007

Este trabajo fue hecho posible por el convenio entre el Banco Mundial y el Ministerio de Sanidad y Asistencia Social; Investigación Operacional PCEE Proyecto VEN/96/002 Línea presupuestaria 021-034

Correspondencia a: Jacobus H. de Waard jacobusdeward@telcel.net.ve 
de TBC pulmonar en individuos sobre 15 años de edad, provenientes de Caracas, Venezuela, y discute la utilidad de la serología para descartar una TBC en pacientes identificados como sintomáticos respiratorios (pacientes SRI) por el Programa de Control de Tuberculosis. Se discute también el valor predictor positivo y negativo de los ensayos, el cual es un parámetro de importancia para la práctica clínica de pruebas de diagnóstico.

\section{Pacientes y Métodos}

Pacientes con tuberculosis pulmonar. Se estudiaron con baciloscopia y cultivo 200 pacientes sobre 15 años de edad, que acudieron consecutivamente entre enero y marzo de 2002, a la consulta del Laboratorio de Tuberculosis del Instituto de Biomedicina, Caracas, Venezuela, identificados como pacientes SRI. Trece resultaron tener baciloscopia positiva/cultivo positivo y siete fueron solamente positivos por cultivo. Tomando al cultivo como la prueba de oro, hubo un total de 20 casos de TBC pulmonar (10\% en este grupo). Todos los aislados fueron identificados como $M$. tuberculosis con pruebas bioquímicas. En la Tabla 1 se resumen los resultados del diagnóstico por baciloscopia y cultivo. En este estudio la sensibilidad de la baciloscopia en el diagnóstico fue de 65\%; (13/20). El cultivo detectó siete pacientes más, un aumento de $53 \%$. Todos los pacientes que resultaron tener baciloscopia positiva también tenían cultivo positivo, lo que permitió asignar a la baciloscopia, para esta serie, una especificidad de $100 \%$.

Pacientes controles. Para determinar la especificidad de los ensayos de ELISA se usaron sueros de dos grupos de pacientes control, tomados entre enero y marzo de 2002. En ambos grupos, todos los pacientes habían recibido la vacuna BCG y tenían prueba de tuberculina positiva (induración de 15 mm o más). Un grupo consistió en 20 personas sanas: 10 estudiantes del sexto año de Medicina y 10 contactos de pacientes tuberculosos. El otro grupo consistió en 20 pacientes SRI y tuberculina positiva, que consultaron el laboratorio para

\section{Tabla 1. Estudio de tuberculosis pulmonar por baciloscopia y cultivo de} expectoración en 200 pacientes sintomáticos respiratorios, Instituto de Biomedicina, Caracas, Venezuela

\begin{tabular}{|lccc|} 
Baciloscopia & Positivo & $\begin{array}{c}\text { Cultivo } \\
\text { Negativo }\end{array}$ & Total \\
\hline Positiva & 13 & 0 & 13 \\
\hline Negativa & 7 & 180 & 187 \\
\hline Total & 20 & 180 & 200 \\
\hline
\end{tabular}

descartar una TBC. El diagnóstico de TBC de estos últimos, a través de dos muestras de esputo, resultó negativo, tanto por baciloscopia como por cultivo.

Serodiagnóstico de tuberculosis. Los sueros de los pacientes tuberculosos fueron obtenidos antes de iniciar el tratamiento anti-TBC mientras que de los pacientes controles fueron tomados el día que se leyó la prueba de tuberculina. Se extrajo a cada uno $5 \mathrm{ml} \mathrm{de}$ sangre venosa en tubos de ensayo estériles. Una vez coaguladas las muestras, se centrifugaron a $2.000 \mathrm{~g}$ durante 10 minutos y se separó el suero a un tubo Eppendorf. Los sueros fueron congelados a $-20{ }^{\circ} \mathrm{C}$ hasta su uso en serodiagnóstico para infección por VIH y TBC. De todos los pacientes en este estudio, se descartó la infección por VIH con la prueba Determine ${ }^{\circledR} \mathrm{HIV}-1 / 2$ de Abbott Laboratories y se determinó la presencia de anticuerpos contra $M$. tuberculosis por medio de dos ensayos de ELISA comerciales, ambos suministrados por Omega Diagnostics LTD. PATHOZYME-TB Complex Plus ${ }^{\circledR}$ que detecta IgG sérica dirigida a las proteínas recombinantes de 38kDa y 16-kDa de $M$. tuberculosis. El kit es específico para el diagnóstico de TBC activa causada por el complejo M. tuberculosis. Pathozyme-Myco IgG ${ }^{\circledR}$ determina el nivel sérico de IgG a dos antígenos, el lipoarabinomanan (LAM) y la proteína recombinante de 38-kDa. El kit detecta infección por especies de micobacterias. Los kits están provistos con sueros de control positivo bajo y alto y se usó la D.O. del control positivo bajo para la interpretación de los resultados.

Análisis estadístico. Los resultados fueron analizados con la prueba de $\chi^{2}$, en un nivel de confianza de $95 \%$, para determinar que sí hay una diferencia en sensibilidad entre las pruebas de diagnóstico. El valor predictor de una prueba depende de la prevalencia de la enfermedad en la población estudiada. Se calcularon los valores predictores positivo y negativo de los ensayos de ELISA usando las siguientes formulas:

$$
\begin{aligned}
& \mathrm{PPV}=\operatorname{Pr}(\mathrm{S}) /[\operatorname{Pr}(\mathrm{S})+(1-\mathrm{Pr})(1-\mathrm{Sp})] \\
& \mathrm{NPV}=[(1-\mathrm{Pr}) \mathrm{Sp}] /[(1-\operatorname{Pr}) \mathrm{Sp}+\operatorname{Pr}(1-\mathrm{S})]
\end{aligned}
$$

Donde Pr es la prevalencia de TBC pulmonar en el grupo de pacientes SRI estudiados, $\mathrm{S}$ es la sensibilidad del ensayo y Sp la especificidad del mismo.

\section{Resultados}

Sensibilidad del serodiagnóstico en los sueros de los pacientes con tuberculosis pulmonar. Los ensayos de ELISA TB Complex Plus ${ }^{\circledR}$ y Myco IgG ${ }^{\circledR}$ diagnosticaron respectivamente 55 y $80 \%$ de los 20 pacientes como seropositivos para TBC. En 15\% de los 
pacientes (3/20) el serodiagnóstico fue negativo con ambos ensayos de ELISA. La sensibilidad de los ensayos de ELISA para pacientes con baciloscopia positiva y pacientes con baciloscopia negativa no fue significativamente diferente $(\mathrm{p}>0,05)$ (Tabla 2).

Especificidad del serodiagnóstico en los sueros de los pacientes de dos grupos de control. En pacientes sanos, TB Complex Plus ${ }^{\circledR}$ y Myco IgG ${ }^{\circledR}$ diagnosticaron, respectivamente, 5 y 30\% de los pacientes como seropositivos para TBC, por lo que la especificidad calculada para los ensayos de ELISA es de 95 y 70\%, respectivamente.

En el otro grupo de control, pacientes SRI, ambas pruebas diagnosticaron, respectivamente, 5 y $10 \%$ de los pacientes como seropositivos para TBC, por lo que la especificidad calculada es de 95 y 90\%, respectivamente.

Al combinar los resultados de ambos grupos de control, la especificidad de ambas pruebas es de $95 \mathrm{y}$ $80 \%$, respectivamente,.

Valores predictores positivo (VPP) y negativo (VPN) de la baciloscopia y del serodiagnóstico en pacientes SRI. En este estudio la prevalencia de TBC pulmonar en los pacientes SRI seleccionada por las normas del programa de control de TBC nacional, fue de 10\% (20 de los 200 pacientes sintomático respiratorio que consultaron el laboratorio tenían una TBC pulmonar activa). Los VPP y VPN para TB Complex Plus ${ }^{\circledR}$ fueron 93 y 68\% respectivamente, Los VPP y VPN de Myco IgG® fueron 81 y $82 \%$. La baciloscopia tuvo un VPP de $100 \%$ y un VPN de $96 \%$.

\section{Discusión}

En este estudio se evaluó la sensibilidad y especificidad de dos ensayos comerciales de ELISA en pacientes con TBC pulmonar, la forma contagiante de TBC, por lo que constituyen pacientes de alto interés en los programas de control. Los ensayos de ELISA evaluados fueron seleccionados para este estudio por su alta sensibilidad y alta especificidad en un estudio comparativo previo donde se evaluaron siete ensayos comerciales $^{7}$.

La sensibilidad de TB Complex Plus ${ }^{\circledR}$ y Myco IgG ${ }^{\circledR}$ fue de 55 y 80\%, respectivamente, lo cual está por encima de la sensibilidad reportada en la literatura. Tres publicaciones que evaluaron estos dos ensayos reportaron una sensibilidad de 42,4 y $47,0 \%$; 40 y $42 \%^{9}$; 16 y $55 \%^{7}$, respectivamente. La mejor sensibilidad en nuestro estudio puede ser basado en mejor respuesta inmunológica por el HLA fenotipo de la población probada ${ }^{3}$ o ser relacionado con la duración
Tabla 2. Sensibilidad de los ensayos de ELISA en pacientes con diagnóstico de tuberculosis pulmonar por baciloscopia y cultivo o por cultivo solo. Instituto de Biomedicina, Caracas, Venezuela

\begin{tabular}{|c|c|c|c|}
\hline ELISA & $\begin{array}{l}\text { BK positiva } \\
n=13\end{array}$ & $\begin{array}{c}\text { BK negativa } \\
n=7\end{array}$ & $\begin{array}{l}\text { Total } \\
n=20\end{array}$ \\
\hline $\begin{array}{l}\text { Pathozyme-TB Complex Plus }{ }^{\circledR} \\
\text { ( } n=\text { seropositivo) }\end{array}$ & $\begin{array}{c}54 \% \\
(n=7)\end{array}$ & $\begin{array}{c}57 \% \\
(n=4)\end{array}$ & $\begin{array}{c}55 \% \\
(n=11)\end{array}$ \\
\hline $\begin{array}{l}\text { Pathozyme-Myco IgG } \mathbb{} \\
(\mathrm{n}=\text { seropositivo) }\end{array}$ & $\begin{array}{c}84 \% \\
(n=11)\end{array}$ & $\begin{array}{c}71 \% \\
(n=5)\end{array}$ & $\begin{array}{c}80 \% \\
(n=16)\end{array}$ \\
\hline
\end{tabular}

de la enfermedad. Los estudios citados fueron hechos en países desarrollados. La duración de la enfermedad era, probablemente, más larga en los pacientes de este estudio, aumentando así la oportunidad de respuesta humoral a la enfermedad ${ }^{10}$.

La especificidad es otro parámetro que determina la calidad de un método de diagnóstico; en este estudio alcanzó a 95\% para TB Complex Plus ${ }^{\circledR}$, concordante con la especificidad encontrada en otros estudios: $97 \%^{7}$ y $96 \%{ }^{9}$. Para Myco IgG® se ha reportado en la literatura una especificidad de $89^{8}$ y $96 \%{ }^{9}$, lo que concuerda con la especificidad de $90 \%$ observada por los autores en pacientes SRI con tuberculina positiva. Sin embargo, en nuestras manos este ensayo tuvo una baja especificidad (70\%) en pacientes sanos con tuberculina positiva Un posible explicación puede ser la presencia de anticuerpos residuales por una infección reciente con $M$. tuberculosis. El grupo elegido consistió de 10 personas que eran contactos de pacientes tuberculosos, entonces pacientes recién infectados y 10 estudiantes del sexto año de Medicina en práctica desde hacía un año en el hospital, donde posiblemente se infectaron. En el grupo de pacientes controles SRI sin confirmación bacteriológica de TBC, la infección pudo haber sido adquirida durante el transcurso de la vida, por lo que, en general, han tenido más tiempo infectados y la reacción humoral por antígenos de TBC se ha debilitado o se ha extinguido, lo cual eleva la especificidad de los ensayos en este grupo.

En esta población, seleccionada por las normas del programa de control de TBC nacional, la prevalencia de TBC pulmonar fue de 10\% (20 de los 200 pacientes sintomático respiratorio tenían una tuberculosis pulmonar activa). TB Complex Plus ${ }^{\circledR}$ con una especificidad promedio de 95\%, sobrediagnosticará 9 pacientes (falsos positivos) y Myco IgG ${ }^{\circledR}$ con una especificidad promedio de $80 \%$, sobrediagnosticará 36 pacientes (falsos positivos) en este grupo. Aunque la sensibilidad de la baciloscopia es baja, en este estudio tuvo una especificidad de 100\%, un VPP de 100\% y VPN de 
96\%, por lo que resultó superior al ensayo de ELISA. Eso indica que la baciloscopia es una herramienta de diagnóstico más eficiente para descartar TBC en pacientes SRI.

En la práctica clínica, el valor de una prueba de diagnóstico depende también de su VPP y VPN. La sensibilidad y la especificidad de una prueba, teóricamente, son valores fijos pero los valores predictores de una prueba varían con la prevalencia de la enfermedad en el grupo de pacientes en estudio. Basado en la prevalencia de TBC pulmonar de $10 \%$ en nuestro grupo de pacientes SRI, ambos ensayos de ELISA pueden ser útiles como pruebas complementarias para confirmar la enfermedad en aquellos pacientes SRI con alta sospecha clínica de TBC pulmonar (radiología compatible, PPD positivo, contacto) pero baciloscopia negativa. El VPP para TB Complex Plus ${ }^{\circledR}(93 \%)$ permite confirmar la TBC. El VPN de Myco IgG® $(82 \%)$ permite descartar con alta seguridad la TBC en este tipo de paciente. El uso potencial del ensayo de ELISA es en pacientes incapaces de producir un esputo adecuado, es decir, en pacientes pauci-bacilares y baciloscopia negativa o con formas de TBC extra-pulmonar. En este estudio se incluyeron pacientes con baciloscopia negativa pero cultivo positivo. A pesar que el número de pacientes pauci-bacilares fue bajo (siete pacientes), la sensibilidad de los ensayo de ELISA no fue significativamente diferente en pacientes con baciloscopia positiva o negativa (Tabla 2). En pacientes altamente sospechosos y con baciloscopia negativa, un resultado negativo de Myco IgG ${ }^{\circledR}$ descarta con alta seguridad una TBC pulmonar activa y un resultado positivo de TB Complex Plus ${ }^{\circledR}$ puede ser de apoyo en la decisión clínica para iniciar tratamiento antes que el cultivo confirme la TBC pulmonar.

Agradecimientos. Agradecemos el apoyo de la Comisión Europea RDG (INCO-DEV Programa; proyecto no. ICA4CT-2001-10087). J.H. de W es miembro de RELACTB (Tuberculosis Network for Latin America and the Caribbean).

\section{Resumen}

Dos pruebas serológicas, Pathozyme-TB complex plus ${ }^{\circledR}$ y Pathozyme-Myco IgG ${ }^{\circledR}$ fueron evaluadas por su sensibilidad y especificidad con suero de pacientes, no infectados por VIH, con tuberculosis (TBC) pulmonar y personas sanas con la prueba de tuberculina positiva. La sensibilidad y la especificidad de ambas pruebas fueron de 50 y $80 \%$, 95 y $80 \%$, respectivamente. Con una prevalencia de TBC pulmonar de $10 \%$ entre los pacientes que consultan nuestro laboratorio, los valores predictores positivos (VPP) para ambos ensayos fueron de 92 y $80 \%$, respectivamente, mientras que los valores predictores negativos (VPN) fueron de 68 y $80 \%$. En el mismo grupo de estudio, la baciloscopia tuvo un VPP de $100 \%$ y un VPN de $97 \%$. Se concluyó que la baciloscopia es una mejor herramienta diagnóstica para descartar TBC en pacientes. Sin embargo, debido a los altos VPN y VPP de las pruebas de ensayo de ELISA, éstas pueden resultar útiles como pruebas complementarias para excluir o confirmar la enfermedad en determinados pacientes, con baciloscopia negativa y alta sospecha clínica de TBC.

\section{Referencias}

1.- Kim T C, Blackman R S, Heatwole K M, Kim T, Rochester D F. Acid-fast bacilli in sputum smears of patients with pulmonary tuberculosis. Prevalence and significance of negative smears pretreatment and positive smears post-treatment. Am Rev Respir Dis 1984; 129: 264-8.

2.- Levy H, Feldman C, Sacho H, van der Meulen H, Kallenbach J, Koornhof H. A reevaluation of sputum microscopy and culture in the diagnosis of pulmonary tuberculosis. Chest 1989; 95: 1193-7.

3.- Bothamley G H. Serological diagnosis of tuberculosis. Eur Respir J Suppl 1995; 20 : 676s-688s. Review.

4.- Chan E D, Heifets L, Iseman M D.
Immunologic diagnosis of tuberculosis: a review. Tuber Lung Dis 2000; 80: 131-40. Review.

5.- Kassa-Kelembho E, Kassa E, Zandanga G, Service Y B, Ignaleamoko A, Talarmin A. Poor performance of a novel serological test for diagnosis of pulmonary tuberculosis in Bangui, Central African Republic. Clin Vaccine Immunol 2006; 13: 702-3.

6.- Ongut G, Ogunc D, Gunseren F, Ogus C, Donmez L, Colak D, et al. Evaluation of the ICT Tuberculosis test for the routine diagnosis of tuberculosis. BMC Infect Dis 2006; 6: 37.

7.- Pottumarthy S, Wells V C, Morris A J. A comparison of seven tests for serological diagnosis of tuberculosis. J Clin Microbiol 2000; 38: 2227-31.
8.- Kondo A, Oketani N, Maruyama M, Saito Y, Miyao H, Ohno M, et al. Serological diagnosis of pulmonary tuberculosis and nontuberculous pulmonary mycobacteriosis. Kekkaku 2001; 76: 603-14.

9.- López, et al. Conference on Global Lung Health and the 1996 Annual Meeting of the International Union Against Tuberculosis and Lung Disease (IUATLD). Paris, France, 2-5 October 1996. Tubercle and Lung Disease 1996; 77 (Suppl 2): 119 (abstract).

10.- McConkey S J, Youssef F G, Azem E, Frenck R W, Weil G J. Evaluation of a rapid-format antibody test and the tuberculin skin test for diagnosis of tuberculosis in two contrasting endemic settings. Int J Tuberc Lung Dis 2002; 6: $246-52$ 